\title{
Evaluación de la carga laboral en residentes Chilenos de especialidades y subespecialidades médicas
}

\author{
Luis Antonio Díaz ${ }^{1}$, Juan Pablo Arab ', Petre Cotoras ' , Daniela Véliz ${ }^{1}$, Marcela Bitrán ${ }^{1}$, Viviana Rojas ${ }^{1}$, Marcelo Lopetegui ${ }^{1}$, \\ Patricio Torres ${ }^{1}$, Matías González ${ }^{1}$, Trinidad Hoyl ${ }^{* *}$, Arnoldo Riquelme ${ }^{1^{*}}$
}

\begin{abstract}
Introducción: La sobrecarga laboral horaria es un importante problema en los programas de especialización médica. Se asocia a mayor prevalencia de depresión, burnout, deserción, e impacta en la calidad de vida de los residentes. Nuestro objetivo fue cuantificar las horas semanales de carga laboral horaria en residentes de especialidad y subespecialidad de la Pontificia Universidad Católica de Chile (PUC). Métodos: Se realizó una encuesta electrónica a los residentes de especialidad y subespecialidad de la Pontificia Universidad Católica de Chile (2013). Esta incluyó autorreporte de horas semanales trabajadas (jornada habitual, turnos presenciales y turnos de llamada). Para el análisis se consideraron solo las horas presenciales (jornada habitual y turnos presenciales). Resultados: se obtuvieron 415 respuestas (tasa de respuesta 86\%). Los residentes de especialidad reportaron una jornada de $52,8 \pm 20,3$ horas semanales más un promedio de 22,6 $\pm 22,5$ horas de turnos presenciales semanales. Los residentes de programas de subespecialidad reportaron una jornada de 55,9 $\pm 22,2$ horas semanales más un promedio de $23 \pm 3,9$ horas de turnos presenciales semanales. De los 53 programas evaluados, el 22,6\% sobrepasó el límite recomendado por la Dirección de Postgrado PUC (80 horas semanales). Cinco programas reportaron 90-100 horas semanales (todas especialidades o subespecialidades quirúrgicas) y 7 programas reportaron $80-90$ horas semanales ( 3 subespecialidades médicas, 1 subespecialidad quirúrgica, 2 especialidades médicas y 1 especialidad quirúrgica). Conclusión: los residentes de especialidades y subespecialidades médicas en la Pontificia Universidad Católica de Chile están sometidos a una carga horaria significativa. Los programas quirúrgicos presentaron la mayor sobrecarga horaria presencial, con un promedio mayor al recomendado a nivel internacional.
\end{abstract}

Palabras clave: Residentes; medicina; especialidad; subespecialidad; ciencias médicas

Abstract: Introduction: Residents work hours' overload constitutes an important problem in medical postgraduate residency programs. It has been associated to an increased prevalence of depression, burnout, attrition and affects quality of life of residents. The aim of our study was to quantify the duty hours in residents of specialty and subspecialty at the Pontificia Universidad Católica de Chile (PUC). Methods: An electronic survey was performed to residents of specialty and subspecialty at PUC (2013). It included an auto report of the duty hours worked in a week (regular working day, night shifts and on call shifts). We only included for analysis the face hours (regular working day and night shifts). Results: 415 surveys were answered (response rate $86 \%$ ). Specialty residents reported $52.8 \pm 20.3$ regular working hours per week plus an average of $22.6 \pm 22.5$ hours of night shifts. Subspecialty residents reported $55.9 \pm 22.2$ regular working hours per week plus an average of $23 \pm 3.9$ hours of night shifts. From the 53 evaluated programs, $22.6 \%$ exceeded the limit recommended in the PUC Postgraduate Office ( 80 hours per week). Five programs reported 90-100 hours per week (all were surgical specialties and subspecialties) and 7 programs reported 80-90 hours per week (3 medical subspecialties, 1 surgical subspecialty, 2 medical specialties and 1 surgical specialty). Conclusion: The residents of specialty and subspecialty from medical postgraduate programs at PUC are subject to a significant workload. The surgical programs present the highest time workload, with a greater average than the internationally recommended.

Keywords: Residents; medicine; specialty; subspecialty; medical sciences

Fecha de envío: 17 de junio de 2016 - Fecha de aceptación: 30 de agosto de 2016

1) Escuela de Medicina, Pontificia Universidad Católica de Chile. Santiago de Chile.

*Autores de correspondencia: a.riquelme.perez@gmail.com / mthoyl@med.puc.cl 


\section{Introducción}

La etapa de la especialización médica es una dimensión esencial en la transformación del médico en experto sobre algún área del conocimiento médico. Este periodo puede resultar física, emocional e intelectualmente demandante en la medida que el residente gana experiencia y desarrolla sus habilidades clínicas, ya que se le exige ser responsable de una importante carga académica y laboral. (Passalacqua et al. 2012; Hochberg et al. 2013)

La sobrecarga horaria es un problema ampliamente descrito en programas de especialización médica en Estados Unidos (EE. UU.) y Europa. Es considerada un problema relevante, ya que puede llevar a fatiga, burnout, menor destreza quirúrgica, menor realización personal, menor cantidad de horas de sueño y mayor deserción, lo que puede dañar la salud de los residentes a largo plazo (Bannai et al. 2014; Mansukhani et al. 2012; Harris et al. 2014). Adicionalmente, podría perjudicar a los pacientes como consecuencia de mayores tasas de errores médicos y menor satisfacción con la atención recibida. (Lafreniere et al. 2016; Ouyang et al. 2016; Prins et al. 2009)

A pesar de la vasta investigación en EE.UU. y Europa, aún no existe consenso internacional respecto a los límites máximos de carga laboral en los diferentes programas de especialización. La Accreditation Council for Graduate Medical Education (ACGME) es la organización responsable de la acreditación de los programas de especialización médica en EE. UU. Define las horas de trabajo como "la cantidad de tiempo que el residente gasta en la experiencia clínica y en educación en su especialidad o subespecialidad" (ACGME 2016). En particular, refiere que "experiencia clínica y educación incluyen todas las actividades clínicas y académicas de un programa, lo que incluye cuidado de pacientes (hospitalizado y ambulatorio), tareas administrativas relativas al cuidado de pacientes, las entregas de turno, el tiempo en casa durante turnos de llamado y actividades programadas como congresos, y no incluye el tiempo de estudio y preparación afuera del recinto educacional" (ACGME 2016). Por otro lado, regula aspectos tales como criterios de inclusión a programas de residencias, características específicas de los programas, tipo de conocimientos que deben ser manejados, métodos de evaluación, manejo del estrés y cansancio, horas de trabajo, entre otras (ACGME 2016).

La ACGME ha llevado a cabo importantes reformas en cuanto a la restricción horaria en los programas de especialidades médicas. Específicamente, estableció en el año 2003 que la cantidad de horas de trabajo debe estar limitada a 80 horas promedio a la semana, incluyendo actividades diurnas, nocturnas y de llamado, lo que actualmente está publicado en la sección VI de "Common Program Requirements" (ACGME 2016; Nasca et al. 2010). Solo permite extender un $10 \%$ este límite en situaciones excepcionales como en casos de pacientes severamente inestables, eventos docentes relevantes para la formación o atención a necesidades especiales de un paciente o su familia. Por otra parte, la ACGME también recomienda que los programas de especialización entreguen al residente herramientas suficientes para reconocer signos de fatiga y deprivación de sueño, y estrategias para combatirlos. (ACGME 2016). Existen otras iniciativas internacionales como la desarrollada en Quebec (Canadá), el 2012 donde se estableció un límite máximo de 16 horas continuas de trabajo para los residentes (Dussault et al. 2014), y otras ciudades canadienses que han establecido un límite de 89-90 horas semanales (Materson et al. 2014). Adicionalmente, organizaciones como el Royal College of Physicians and Surgeons of Canadá (RCPSC) y su programa CanMEDS, han establecido recomendaciones sobre la carga laboral horaria, con el fin de que logran un desempeño acorde a lo esperado en sus residentes, sin deteriorar la calidad de vida de ellos (RCSPC, 2016).

Diversos estudios han intentado medir el impacto de la reforma de carga laboral impulsada por la ACGME en el 2011; en ellos no se observó una disminución en la tasa de burnout, fatiga, errores médicos ni mortalidad hospitalaria (Hutter et al. 2006; Sneider et al. 2009; Ahmed et al. 2014; Jamal et al. 2014). A diferencia de Norteamérica, en Chile y Latinoamérica hay escasos trabajos que evalúen la cantidad de horas de carga asistencial a los que están sometidos los residentes de los diferentes programas de especialización y subespecialización médica.

Dada la ausencia de información sobre la sobrecarga horaria en residentes a nivel nacional, el objetivo de este trabajo fue cuantificar la carga horaria laboral semanal en residentes de especialidad y subespecialidad en la Pontificia Universidad Católica de Chile (PUC).

\section{Métodos}

El año 2013 se aplicó una encuesta de calidad de vida y aspectos académicos por parte de la Dirección de Postgrado de la Escuela de Medicina de la Pontificia Universidad Católica de Chile a todos los residentes tanto de especialidades, como de subespecialidades médicas de nuestra institución. La encuesta incluyó autorreportes respecto a las horas promedio dedicadas a trabajo semanal, turno presencial, turnos de llamada semanal, días promedio completamente libres en un mes, número máximo de horas continuas trabajadas en los últimos 6 meses, horas de actividades docentes formales programadas por semana, porcentaje de actividad clínica bajo supervisión y horas de estudio independiente promedio en una semana. Esta encuesta fue contestada de forma electrónica, mediante un formulario enviado de manera personal a cada residente por correo electrónico. 
Para el análisis se consideraron solo las horas presenciales (jornada habitual y turnos presenciales). El análisis consideró 24 programas de especialidad y 29 de subespecialidad. Los programas de especialidad, se organizaron en 4 subgrupos correspondientes a programas Médicos (166 residentes), Médico-quirúrgicos (30 residentes), Quirúrgicos (94 residentes) y Especialidades de Diagnóstico y Gestión (42 residentes) (Tabla 1). Los programas de subespecialidad se subdividieron en Médicos (61 residentes) y Quirúrgicos (22 residentes) (Tabla 2).
El uso de los datos fue de manera anónima y los analistas fueron externos a la Dirección de Postgrado y los programas de formación. Además, se obtuvo la aprobación del Comité de Ética de la Pontificia Universidad Católica de Chile, quienes otorgaron una dispensa de consentimiento.

Las datos continuos fueron descritos mediante promedio y desviación estándar de acuerdo con la distribución de los datos. Los datos nominales fueron descritos mediante porcentajes. Los análisis estadísticos fueron realizados mediante el software IBM SPSS versión 22,0 (Armonk, NY: IBM Corp.).

Tabla 1.- Programas de especialidades.

\begin{tabular}{|l|l|l|l|}
\hline \multicolumn{1}{|c|}{ Médicas (n=166) } & \multicolumn{1}{|c|}{ Quirúrgicas (n=94) } & \multicolumn{1}{c|}{ Médico-Quirúrgicas (n=30) } & \multicolumn{1}{c|}{ Diagnóstico y Gestión (n=42) } \\
\hline - Medicina Familiar (mención adulto) & - Anestesiología & - Dermatología & - Anatomía Patológica \\
- Medicina Familiar (mención niño) & - Cirugía general & - Oftalmología & - Laboratorio Clínico \\
- Medicina Interna & - Neurocirugía & - Otorrinolaringología & - Medicina Nuclear \\
- Medicina Urgencia & - Obstetricia y Ginecología & & - Radiología \\
- Neurología & - Traumatología y & & - Salud Pública \\
- Neurología Pediátrica & - Urología & & \\
- Nutrición y Diabetes & & & \\
- Pediatría & & & \\
- Psiquiatría & & & \\
- Radio-oncología & & & \\
\hline
\end{tabular}

Tabla 2.- Programas de subespecialidades

\begin{tabular}{|l|l|}
\hline \multicolumn{1}{|c|}{ Médicas (n=61) } & \multicolumn{1}{c|}{ Quirúrgicas (n=22) } \\
\hline - Cardiología & \\
- Cardiología Pediátrica & \\
- Endocrinología del Adulto & \\
- Endocrinología Pediátrica & - Anestesia Cardiovascular \\
- Enfermedades Respiratorias Pediátrica & - Anestesia Regional y Analgesia Perioperatoria \\
- Enfermedades Respiratorias del Adulto & - Cirugía cardiovascular \\
- Gastroenterología del Adulto & - Cirugía digestiva \\
- Gastroenterología y Nutrición Pediátrica & - Cirugía Oncológica de Cabeza y Cuello \\
- Geriatría & - Cirugía Plástica y Reconstructiva \\
- Hematología del Adulto & - Cirugía Vascular Periférica \\
- Infectología Pediátrica & - Ginecología oncológica \\
- Medicina Intensiva Adultos & - Materno-fetal \\
- Inmunología Clínica y Reumatología & \\
- Medicina Intensiva Infantil & \\
- Nefrología & \\
- Neonatología & \\
- Oncología Médica & \\
\hline
\end{tabular}

\section{Resultados}

Cuatrocientos quince residentes de especialidades y subespecialidades respondieron la encuesta durante los meses de septiembre, octubre y noviembre del 2013. De estos, 332 eran residentes de especialidades y 83 de subespecialidades. La tasa de respuesta fue $86 \%$. El promedio de edad fue $29,5 \pm 3,2$ años. Hubo 211 residentes de sexo femenino $(50,8 \%)$ y 204 de sexo masculino $(49,2 \%)$.
Con respecto a las horas de trabajo, en promedio los residentes de programas de especialidad reportaron una carga horaria diurna de $53 \pm 10$ horas semanales más un promedio de $22 \pm 12,5$ horas de turnos presenciales semanales. En la Figura 1 se ilustra en detalle las horas de trabajo presencial (carga horaria diurna y carga horaria diurna más turnos) en los distintos subgrupos. 


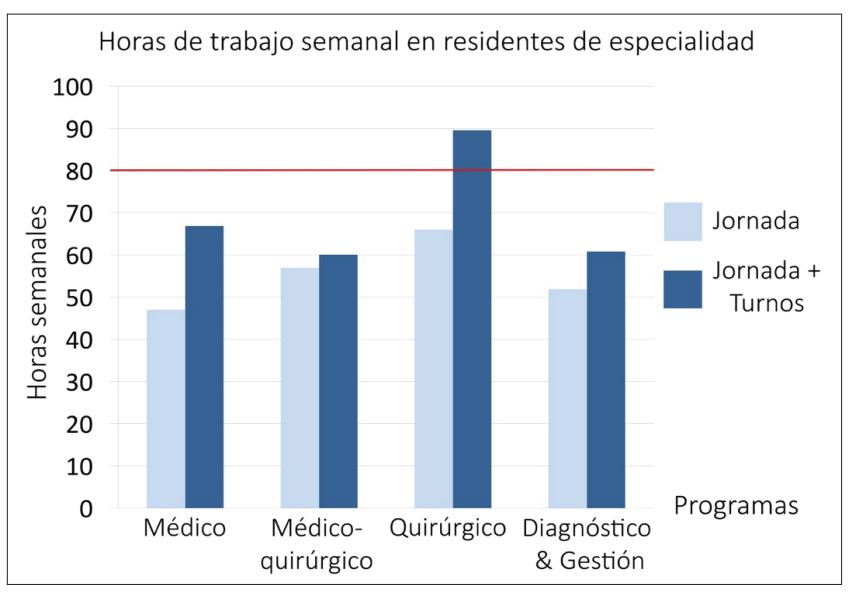

Figura 1.- Promedio de carga horaria laboral semanal en residentes de especialidad, separadas entre carga horaria diurna y carga horaria diurna más turnos presenciales.

Por su parte, los residentes de programas de subespecialidad reportaron una carga horaria diurna de $56 \pm 11$ horas semanales promedio, más un promedio de $23 \pm 4$ horas de turnos presenciales semanales. En la Figura 2 se observan las diferencias entre subespecialidades médicas y quirúrgicas.

Horas de trabajo semanal en residentes de subespecialidad

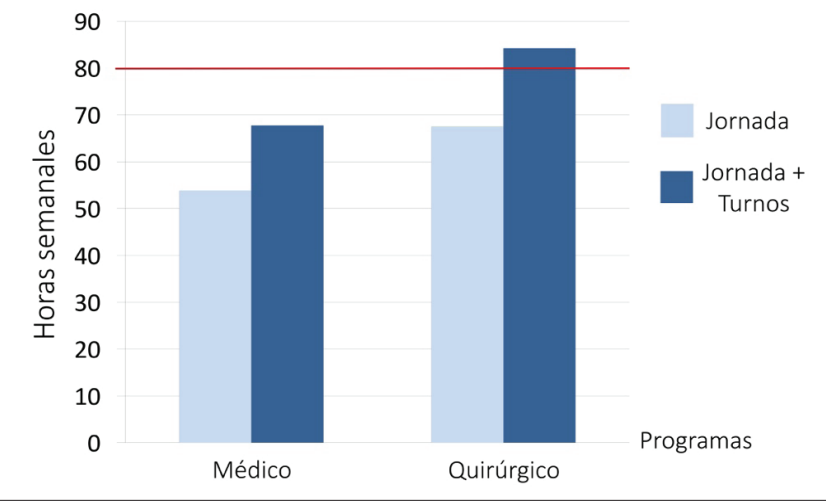

Figura 2.- Promedio de carga horaria laboral en subespecialidades de la Pontificia Universidad Católica de Chile, divididas entre carga horaria diurna y carga horaria diurna más turnos presenciales.

De los 53 programas de especialidades y subespecialidades estudiados, 12 programas (22,6\% del total) sobrepasaron el límite de 80 horas semanales de trabajo presencial recomendado por la Dirección de Postgrado PUC y por la ACGME. De estos, cinco programas reportaron entre 90 y 100 horas semanales de jornada habitual más turnos presenciales (correspondientes a especialidades y subespecialidades quirúrgicas) y 7 programas reportaron entre 80 y 90 horas semanales (3 subespecialidades médicas, 1 subespecialidad quirúrgica, 2 especialidades médicas y 1 especialidad quirúrgica). Además, 8 programas reportaron entre 70 y 80 horas semanales ( 3 subespecialidades médicas, 1 subespecialidad quirúrgica 2 especialidades médicas, 1 especialidad de diagnóstico, 1 especialidad quirúrgica) y 33 programas reportaron $<70$ horas semanales. En la Figura, 3 se ilustra la distribución de especialidades y subespecialidades, agrupados en rangos de acuerdo con la cantidad de horas trabajadas semanalmente.

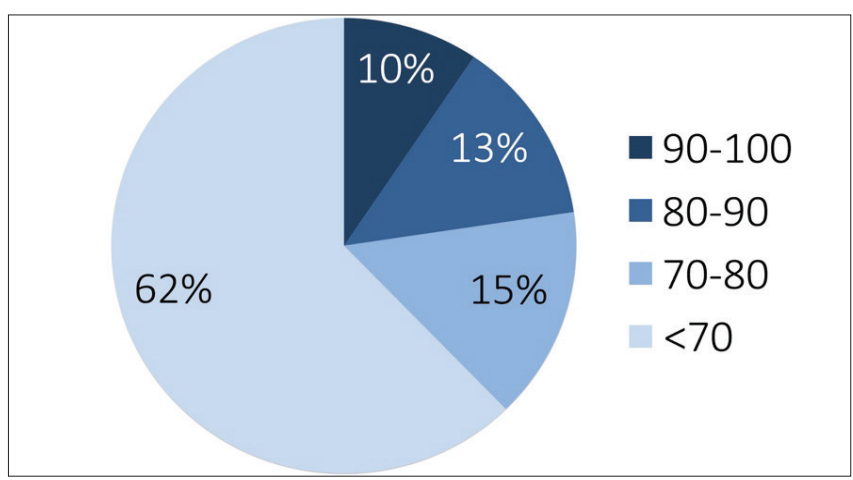

Figura 3.- Distribución de programas de especialidad de acuerdo a la cantidad de horas de trabajo presencial (jornada habitual más turnos presenciales) semanales.

\section{Discusión}

La carga horaria laboral de los médicos en formación en programas de especialización médica es un factor relevante, que influye en varios aspectos de su diario vivir $\mathrm{y}$, por ello, podría condicionar su calidad de vida, el aprendizaje y su desempeño con el paciente (de Beer et al. 2016).

El presente trabajo muestra que la carga horaria laboral semanal de residentes de un $23 \%$ de los programas de especialidad y subespecialidad de la Pontificia Universidad Católica de Chile, está por sobre el límite máximo de horas recomendado internacionalmente por la ACGME. Estos son, en su mayoría, programas de especialidades o subespecialidades quirúrgicas.

La ACGME ha hecho importantes esfuerzos en regular la cantidad de horas promedio semanales, horas máximas seguidas trabajando y tiempo libre entre jornadas laborales, fijando recomendaciones sobre las horas promedio semanales totales en 80 horas (incluyendo trabajo fuera del recinto asistencial). En EE. UU. se ha estudiado el efecto de la implementación de estas reglas en la reforma del 2003 y 2011, encontrando resultados contradictorios respecto del impacto de la restricción de horas en hospitales docentes. Algunos han demostrado mejoras, como el aumento en realización personal y menor cansancio emocional (Barrack et al. 2006). Otros estudios primarios y revisiones sistemáticas no han evidenciado una disminución la mortalidad global ni la percepción de los pacientes sobre la calidad de atención médica tras instaurar las reformas. Tampoco 
variaron las tasas de fatiga, burnout, horas de sueño, especialmente en los residentes de especialidades quirúrgicas (Hutter et al. 2006; Sneider et al. 2009; Ahmed et al. 2014; Jamal et al. 2014; Ripp et al. 2015; Bolster et al. 2015).

Aunque los estudios previamente señalados no avalan la disminución de carga horaria a 80 horas semanales, estudios previos han demostrado que la sobrecarga laboral horaria genera un 464\% más de errores diagnósticos graves en la unidad de paciente crítico, $168 \%$ más de colisiones en vehículo posturno y $73 \%$ más riesgo de accidentes corto punzantes (Landrigan et al. 2004; Barger et al. 2005; Ayas et al. 2006). Así, tomando estas evidencias, resulta claro que al menos hacen falta estudios que utilicen puntos de corte más bajos de horas laborales promedio semanales para evaluar su real impacto en el cuidado de los pacientes y la calidad de vida de los residentes.

Dentro de las posibles estrategias para disminuir la sobrecarga laboral de los residentes, se podría proponer: fragmentar el horario de ingreso de los residentes con el fin de contar con más residentes en horario con mayor demanda y menos residentes en horarios con menor demanda; también se puede intentar disminuir el número de turnos totales por residente. Otra posible estrategia podría ser otorgar la salida inmediatamente después de terminado el turno, lo que implicaría en especialidades hospitalarias reorganizar los sistemas de atención a pacientes, a fin de cubrir los pacientes del residente posturno.

Ahora bien, la reducción de la sobrecarga horaria no es la única estrategia que se asocia a disminuir el error médico. De hecho, una revisión sistemática citada previamente concluyó que enfocarse en disminuir las horas de trabajo por sí solo, no ha demostrado mejoras en el cuidado del paciente ni en el bienestar de los residentes (Bolster et al. 2015). En otro estudio se identificaron ocho tipos de intervenciones, de las cuales 4 demostraron disminuir el error médico: cambios en los horarios de trabajo, modos de la educación, conciliación farmacoterapeútica y la incorporación de protocolos y guías (Manias et al. 2012). Sin embargo, la conclusión del estudio fue cautelosa, debido a la diversidad de diseños de estudios incorporados y ausencia de grupos control.

La recomendación de restricción horaria en residentes aún constituye un tema controversial. Una restricción de horas muy estricta como la recomendada en algunos países (36, 48 ó 56 horas/semana máximo) claramente acorta las oportunidades de formación docente y la experiencia clínica, lo que nos plantea la necesidad de prolongar los programas. Por otro lado, la desregulación absoluta puede dejar abierta la puerta para que haya carga excesiva de trabajo, ya sea por necesidades asistenciales del sistema o por entusiasmo irresponsable de docentes y residentes, con el riesgo potencial de dañar el bienestar del residente y disminuir la calidad de la atención del paciente.

En nuestra realidad, se ha establecido una recomendación general de no sobrepasar las 80 horas/semana en promedio en 1 mes. Nos parece que esta recomendación se ajusta bastante a nuestra realidad, ya que en la práctica equivale a un horario de hasta 11 horas de lunes a viernes, más 4 horas de sábado y 5 turnos al mes (incluyendo 2 fines de semana o festivos). Estamos conscientes de que un modelo estricto de restricción horaria es inviable para todos los programas, pues especialmente en los programas quirúrgicos, hay oportunidades de aprendizaje que se dan en un momento único y son de gran valor.

Por lo tanto, más importante que solo basar las recomendaciones en el horario de trabajo, planteamos que se debe velar por que en los programas de residencia haya: oportunidades de aprendizaje en número y calidad adecuadas, en equilibrio con una calidad de vida razonable, un ambiente educacional positivo y de respeto mutuo, estrategias para detectar a los residentes con estrés y burnout, normativas generales de bienestar para residentes, y medidas determinantes de la seguridad en la atención del paciente.

Una mirada complementaria a las medidas de reducción del burnout y el estrés entre residentes es la potenciación del bienestar subjetivo y el autocuidado. A este respecto, existe un aumento reciente en el reporte de programas e intervenciones que propenden a estos objetivos (Shanafelt et al., 2005; Eckleberry-Hunt et al., 2009; Cedfeldt et al., 2010; Robiner et al., 2013; Devens et al., 2012). El aumento de bienestar está asociado a múltiples consecuencias deseables, entre ellas, a un mayor involucramiento en el trabajo (engagement). Este ha sido definido como un estado mental positivo y satisfactorio frente al trabajo, como resultado de altos niveles de vigor, dedicación y absorción (Schaufeli et al., 2002). El desarrollo de programas destinados a aumentar el engagement, en consecuencia, podría contribuir al bienestar y profesionalismo de los residentes durante su formación, contrarrestando los efectos deletéreos de la sobrecarga laboral.

Nos parece que este trabajo contribuye a realizar un diagnóstico sobre la carga laboral en residentes de especialidades y subespecialidades en la Pontificia Universidad Católica de Chile. Estos datos permitirán a nuestra institución establecer medidas para regulary apoyar los diversos programas de formación en el cumplimiento de estos horarios, con el fin de mejorar la calidad de vida de los residentes y favorecer el uso del tiempo para otras actividades tales como talleres complementarios, actividades de investigación, entrenamiento en modelos simulados, entre otros. 
Entre las limitaciones más importantes de este estudio destaca la recolección de datos, realizada en base a autorreportes electrónicos de las horas semanales promedio en los últimos 6 meses. Este método podría aumentar las horas de trabajo reportadas en relación con las reales, ya que los residentes pueden tener una percepción de horas trabajadas mayor a la real. No obstante, el autorreporte también puede asociarse con sentimientos de miedo, presión y estrés por parte de los residentes al momento de evaluar el programa de especialización de manera no anónima, lo que conduciría a un sub-reporte de las horas trabajadas (Carpenter et al. 2006). Estas limitantes han sido evaluadas previamente en un estudio prospectivo (Todd et al. 2010), donde se comparó el autorreporte de horas trabajadas con un sistema de medición electrónica que marcaba entradas y salidas del hospital. Se concluyó que la tasa de reporte de sobrecarga laboral fue similar en ambos métodos, generando controversia sobre la validez del autorreporte. Por otro lado, el considerar como periodo los últimos 6 meses puede considerar horarios de rotaciones muy heterogéneas, con cargas horarias laborales en consecuencia diferentes.

En este análisis tampoco fueron consideradas las horas semanales en turno de llamado. Dado que estos turnos pueden constituir una importante carga laboral horaria, especialmente en algunas subespecialidades, la ACGME también ha elaborado recomendaciones específicas para estas. Las horas en turno de llamado deberán ser objeto de análisis y regulación en futuros estudios de carga laboral.

Nuestra institución ha intentado mejorar continuamente la calidad de formación de sus residentes. En este sentido, ha implementado paulatinamente el programa CanMEDS de la RCPSC, alcanzando su acreditación el 2016. Con respecto a la carga horaria laboral, la RCPSC plantea 5 puntos principales: (1) los residentes tienen roles interrelacionados como aprendices y proveedores de salud, (2) los residentes son proveedores de salud vitales en un sistema de salud y son colectivamente responsables en la cobertura del cuidado a los pacientes 24/7, (3) Los periodos de trabajo continuo de 24 horas o más debieran ser evitados, (4) son necesarios esfuerzos para minimizar riesgos y mejorar la seguridad, lo cual no puede conseguirse solo abordando el tema de la carga laboral de los residentes, y (5) dada la importante variación en las necesidades de entrenamiento de los residentes, se necesita un modelo riguroso y adaptable de carga horaria laboral. Aunque no plantean un número absoluto aplicable a todos los programas, rotaciones y residentes de diferentes especialidades ponen énfasis en las diferentes necesidades de cada programa y que no solo se debe abarcar el problema desde el punto de vista de la carga laboral. (RCPSC 2016).
Finalmente, es necesario conocer la carga laboral en residentes de otras instituciones, para poder abordar este tópico a nivel nacional y generar regulaciones a largo plazo, puesto que en la actualidad no existe ningún organismo nacional que aborde este tópico. Esperamos poder contribuir también con otros países latinoamericanos para el diagnóstico y enfrentamiento de este problema.

\section{Conclusiones}

Los residentes de especialidades y subespecialidades médicas están sometidos a una carga laboral significativa. Los programas de especialidades y subespecialidades quirúrgicas presentaron la mayor sobrecarga laboral presencial, con un promedio mayor al recomendado a nivel internacional.

\section{Referencias}

Accreditation Council for Graduate Medical Education (ACGME) (2016). Duty Hours Overview. Citado el 30 de agosto del 2016. Disponible en: [http://www.acgme.org/Portals/0/PDFs/DutyHoursOverview.pdf].

Accreditation Council for Graduate Medical Education (ACGME) (2016). Common Program Requirements. Citado el 30 de agosto del 2016. Disponible en: https://www.acgme.org/Portals/0/PFAssets/ ProgramRequirements/CPRs_07012016.pdf].

Ahmed N, Devitt KS, Keshet I, Spicer J, Imrie K, Feldman L, Cools-Lartigue J, Kayssi A, Lipsman N, Elmi M, Kulkarni AV, Parshuram C, Mainprize T, Warren RJ, Fata P, Gorman MS, Feinberg S \& Rutka J (2014). A systematic review of the effects of resident duty hour restrictions in surgery: impact on resident wellness, training, and patient outcomes. Annals of surgery 259, 1041-1053.

Ayas NT, Barger LK, Cade BE, Hashimoto DM, Rosner B, Cronin JW, Speizer FE \& Czeisler CA (2006). Extended work duration and the risk of self-reported percutaneous injuries in interns. JAMA 296, 1055-1062.

Baldwin K, Namdari S, Donegan D, Kamath AF \& Mehta S (2011). Early effects of resident work-hour restrictions on patient safety: a systematic review and plea for improved studies. The Journal of bone and joint surgery 93 , e5.

Bannai A \& Tamakoshi A (2014). The association between long working hours and health: a systematic review of epidemiological evidence. Scandinavian journal of work, environment \& health 40, 5-18.

Barger LK, Cade BE, Ayas NT, Cronin JW, Rosner B, Speizer FE \& Czeisler CA; Harvard Work Hours, Health, and Safety Group (2005). Extended work shifts and the risk of motor vehicle crashes among interns. N Engl J Med. 352, 125-134. 
Barrack RL, Miller LS, Sotile WM, Sotile MO \& Rubash HE (2006). Effect of duty hour standards on burnout among orthopaedic surgery residents. Clinical orthopaedics and related research 449, 134-137.

Bolster L \& Rourke L (2015). The Effect of Restricting Residents' Duty Hours on Patient Safety, Resident Well-Being, and Resident Education: An Updated Systematic Review. Journal of graduate medical education 7, 349-363.

Cedfeldt, AS, Bower EA, English C, Grady-Weliky TA, Girard DE \& Choi D (2010). Personal time off and residents' career satisfaction, attitudes and emotions. Medical Education 44, 977-984.

Carpenter RO, Spooner J, Arbogast PG, Tarpley JL, Griffin MR, Lomis KD (2006). Work hours restrictions as an ethical dilemma for residents: descriptive survey of violation types and frequency. CurrSurg 63, 448-455.

de Beer LT, Pienaar J, Rothmann S Jr (2016). Work overload, burnout, and psychological ill-health symptoms: a three-wave mediation model of the employee health impairment process. Anxiety Stress Coping 29, 387-399.

Devens M, Hasnain M, Dudkiewicz B \& Connell KJ (2012). Facilitating resident well-being: a pilot intervention to address stress and teamwork issues on an inpatient service. Fam Med 44, 265-268.

Dussault C, Rousseau MC, Saad N \& Carrier J (2014). 16-hour call duty schedules: the Quebec experience. BMCMedEduc 14, (S1):S10.

Eckleberry-Hunt J, Van Dyke A, Lick D \& Tucciarone J (2009). Changing the Conversation From Burnout to Wellness: Physician Well-being in Residency Training Programs. J Grad Med Educ 1, 225-230.

Fletcher KE, Reed DA \& Arora VM (2011). Patient safety, resident education and resident well-being following implementation of the 2003 ACGME duty hour rules. Journal of general internal medicine 26, 907-919.

Harris JD, Staheli G, LeClere L, Andersone D \& McCormick F (2014). What Effects Have Resident Work-hour Changes Had on Education, Quality of Life, and Safety? A Systematic Review. Clinical orthopaedics and related research 473, 1600-1608.

Hochberg MS, Berman RS, Kalet AL, Zabar SR, Gillespie C, Pachter HL (2013). The stress of residency: recognizing the signs of depression and suicide in you and your fellow residents. American Journal of Surgery 205, 141-146.

Hutter MM, Kellogg KC, Ferguson CM, Abbott WM \& Warshaw AL (2006). The impact of the 80-hour resident workweek on surgical residents and attending surgeons. Annals of surgery 243, 864-875.
Jamal MH, Doi SA, Rousseau M, Edwards M, Rao C, Barendregt JJ, Snell L \& Meterissian S (2012). Systematic review and meta-analysis of the effect of North American working hours restrictions on mortality and morbidity in surgical patients. The British journal of surgery 99, 336-344.

Jamal MH, Wong S \& Whalen TV (2014). Effects of the reduction of surgical residents' work hours and implications for surgical residency programs: a narrative review. BMC medical education 14, Suppl 1:S14.

Lafreniere JP, Rios R, Packer H, Ghazarian S, Wright SM, Levine RB (2016). Burned Out at the Bedside: Patient Perceptions of Physician Burnout in an Internal Medicine Resident Continuity Clinic. Journal of General Internal Medicine 31, 203-208.

Landrigan CP, Rothschild JM, Cronin JW, Kaushal R, Burdick E, Katz JT, Lilly CM, Stone PH, Lockley SW, Bates DW \& Czeisler CA (2004). Effect of reducing interns' work hours on serious medical errors in intensive care units. N Engl J Med 351, 1838-1848.

Manias E, Williams A \& Liew D (2012). Interventions to reduce medication errors in adult intensive care: a systematic review. British journal of clinical pharmacology 74, 411-423.

Mansukhani MP, Kolla BP, Surani S, Varon J \& Ramar K (2012). Sleep deprivation in resident physicians, work hour limitations, and related outcomes: a systematic review of the literature. Postgraduate medicine 124, 241-249.

Masterson M, Shrichand P \& Maniate JM (2014). Resident duty hours in Canada: a survey and national statement. BMC Medical Education 14, S9.

Moonesinghe SR, Lowery J, Shahi N, Millen A \& Beard JD (2011). Impact of reduction in working hours for doctors in training on postgraduate medical education and patients' outcomes: systematic review. BMJ (Clinical research ed.) 342, d1580.

Nasca TJ, Day SH \& Amis ES Jr (2010). ACGME duty hour task force. The new recommendations on duty hours from the ACGME task force. New England Journal of Medicine 363, e3.

Ouyang D, Chen JH, Krishnan G, Hom J, Witteles R, Chi J (2016). Patient Outcomes when Housestaff Exceed 80 Hours per Week. American Journal of Medicine 129, 993-999.e1.

Parshuram CS, Amaral AC, Ferguson ND, Baker GR, Etchells EE, Flintoft V, Granton J, Lingard L, Kirpalani H, Mehta S, Moldofsky H, Scales DC, StewartTE, Willan AR \& Friedrich JO; Canadian Critical Care Trials Group (2015). Patient safety, resident well-being and continuity of care with different resident duty schedules in the intensive care unit: a randomized trial. CMAJ:Canadian Medical Associationjournal 187, 321-329. 
Passalacqua SA, Segrin C (2012). The effect of resident physician stress, burnout, and empathy on patient-centered communication during the long-call shift. Health Commun 27, 449-456.

Prins JT, van der Heijden FM, Hoekstra-Weebers JE, Bakker AB, van de Wiel HB, Jacobs B, Gazendam-Donofrio SM (2009). Burnout, engagement and resident physicians' self-reported errors. Psychology health medicine 14, 654-666.

Rajaram R, Saadat L, Chung J, Dahlke A, Yang AD, Odell DD \& Bilimoria KY (2015). Impact of the 2011 ACGME resident duty hour reform on hospital patient experience and processes-of-care. BMJ Qual Saf 30, 1-9.

Ripp JA, Bellini L, Fallar R, Bazari H, Katz JT, Korenstein D (2015). The impact of duty hours restrictions on job burnout in internal medicine residents: a three-institution comparison study. Academic Medicine 90, 494-499.

Robiner W, Lazear S \& Duffy B (2013). Promoting health behaviours in medical education. Clin Teach 10, 160-164.
Royal College of Physicians and Surgeons of Canada (RCPSC) (2016). Fatigue, risk and excellence: Towards a pan-Canadian consensus on resident duty hours. Citado el 16 de septiembre del 2016. Disponible en: [http://www.royalcollege.ca/rcsite/education-strategy-accreditation/innovations-development/initiatives/resident-duty-hours-e].

Schaufeli W, Salanova M, González-Roma V \& Bakker A (2002). The measurement of engagement and burnout: $A$ two sample confirmatory factor analytic approach. J Happiness Stud. 3, 71-92.

Shanafelt TD, Novotny P, Johnson ME, Zhao X, Steensma DP, Lacy MQ, Rubin J \& Sloan J (2005). The Well-Being and Personal Wellness Promotion Strategies of Medical Oncologists in the North Central Cancer Treatment Group. Oncology 68, 23-32.

Sneider EB, Larkin AC \& Shah SA (2009). Has the 80-hour workweek improved surgical resident education in New England? Journal of surgical education 66, 140-145.

Todd SR, Fahy BN, Paukert JL, Mersinger D, Johnson ML, Bass B (2010). How accurate are self-reported resident duty hours? Journal of surgical education 67, 103-107. 